Journal for ImmunoTherapy of Cancer

\title{
Clinical and molecular characteristics associated with response to therapeutic PD-1/PD-L1 inhibition in advanced Merkel cell carcinoma
}

\author{
Ivelina Spassova (10 , ${ }^{1,2}$ Selma Ugurel (10) , ${ }^{2}$ Linda Kubat, ${ }^{1,2}$ Lisa Zimmer, ${ }^{2}$ \\ Patrick Terheyden (1) , ${ }^{3}$ Annalena Mohr, ${ }^{3}$ Hannah Björn Andtback, ${ }^{4}$ Lisa Villabona, ${ }^{4}$ \\ Ulrike Leiter, ${ }^{5}$ Thomas Eigentler, ${ }^{5}$ Carmen Loquai, ${ }^{6}$ Jessica C Hassel, ${ }^{7,8}$ \\ Thilo Gambichler, ${ }^{9}$ Sebastian Haferkamp, ${ }^{10}$ Peter Mohr, ${ }^{11}$ Claudia Pfoehler, ${ }^{12}$ \\ Lucie Heinzerling, ${ }^{13}$ Ralf Gutzmer, ${ }^{14}$ Jochen S Utikal, ${ }^{8,15}$ Kai Horny, ${ }^{1,8,16}$ \\ Hans-Ulrich Schildhaus, ${ }^{17}$ Daniel Habermann, ${ }^{18}$ Daniel Hoffmann, ${ }^{18}$ \\ Dirk Schadendorf, ${ }^{2,16}$ Jürgen Christian Becker (D) 1,2,8,16
}

\begin{abstract}
To cite: Spassova I, Ugurel S, Kubat L, et al. Clinical and molecular characteristics associated with response to therapeutic PD-1/PD-L1 inhibition in advanced Merkel cell carcinoma. Journal for ImmunoTherapy of Cancer 2022;10:e003198. doi:10.1136/ jitc-2021-003198
\end{abstract}

- Additional supplemental material is published online only. To view, please visit the journal online (http://dx.doi.org/10. 1136/jitc-2021-003198).

IS and SU contributed equally. Accepted 11 December 2021

Check for updates

(c) Author(s) (or their employer(s)) 2022. Re-use permitted under CC BY-NC. No commercial re-use. See rights and permissions. Published by BMJ.

For numbered affiliations see end of article.

Correspondence to Professor Jürgen Christian Becker; j.becker@dkfz.de

\section{ABSTRACT}

Background Based on its viral-associated or UVassociated carcinogenesis, Merkel cell carcinoma (MCC) is a highly immunogenic skin cancer. Thus, clinically evident MCC occurs either in immuno-compromised patients or based on tumor-intrinsic immune escape mechanisms. This notion may explain that although advanced MCC can be effectively restrained by treatment with PD-1/ PD-L1 immune checkpoint inhibitors (ICls), a considerable percentage of patients does not benefit from ICl therapy. Biomarkers predicting $\mathrm{ICI}$ treatment response are currently not available.

Methods The present multicenter retrospective study investigated clinical and molecular characteristics in 114 patients with unresectable MCC at baseline before treatment with $\mathrm{ICl}$ for their association with therapy response (best overall response, BOR). In a subset of 21 patients, pretreatment tumor tissue was analyzed for activation, differentiation and spatial distribution of tumor infiltrating lymphocytes (TIL).

Results Of the 114 patients, $n=74(65 \%)$ achieved disease control (BOR=complete response/partial response/ stable disease) on ICI. A Bayesian cumulative ordinal regression model revealed absence of immunosuppression and a limited number of tumor-involved organ systems was highly associated with a favorable therapy response. Unimpaired overall performance status, high age, normal serum lactate dehydrogenase and normal serum $\mathrm{C}$ reactive protein were moderately associated with disease control. While neither tumor Merkel cell polyomavirus nor tumor PD-L1 status showed a correlation with therapy response, treatment with anti-PD-1 antibodies was associated with a higher probability of disease control than treatment with anti-PD-L1 antibodies. Multiplexed immunohistochemistry demonstrated the predominance of $\mathrm{CD}^{+}$effector and central memory $T$ cells $\left(T_{C M}\right)$ in close proximity to tumor cells in patients with a favorable therapy response. Conclusions Our findings indicate the absence of immunosuppression, a limited number of tumor-affected organs, and a predominance of $\mathrm{CD}^{+} \mathrm{T}_{\mathrm{CM}}$ among $\mathrm{TIL}$, as baseline parameters associated with a favorable response to PD-1/PD-L1 ICl therapy of advanced MCC. These factors should be considered when making treatment decisions in MCC patients.

\section{INTRODUCTION}

Merkel cell carcinoma (MCC) is a rare, highly aggressive neuroendocrine skin cancer. MCC carcinogenesis is associated either with the Merkel cell polyomavirus (MCPyV), predominantly in cases occurring in the northern hemisphere, or with chronic UV-exposure. ${ }^{1}$ MCC is highly immunogenic due to the presence of either MCPyV-derived antigens or UV-associated neoantigens. Thus, clinically manifest advanced MCC is mostly observed in immunocompromised and immunosenescent patients or occurs based on tumorintrinsic immune escape mechanisms. Still, a high therapeutic activity of PD-1/PD-L1 immune checkpoint inhibitors (ICIs) with durable objective responses in about $50 \%$ of patients has been observed. ${ }^{2}$ Despite this major improvement in the therapy outcome of advanced MCC patients, this observation also implies that half of the patients do not experience a long-term benefit from ICI therapy. Clinically applicable predictive biomarkers of ICI therapy response are just starting to emerge: (1) in a trial testing neoadjuvant nivolumab, both pathological complete response (CR) and radiographic tumor regression at the time of surgery were correlated with improved recurrence-free survival, ${ }^{3}$ and (2) Kacew et al reported that a limited disease stage at ICI therapy start was associated with a favorable response. ${ }^{4}$ 
However, the study also showed that, unlike in other cancers, neither tumor mutational burden nor copynumber alterations in MCC tumor tissue predicted ICI therapy outcome. Similarly, a recent study by us characterizing 41 MCC patients receiving PD-1/PD-L1 ICI demonstrated that predictive markers of ICI therapy response established in other cancer entities such as neutrophilto-lymphocyte ratio, metastatic stage and site of the primary were not associated with ICI response in MCC. ${ }^{5}$ However, our comprehensive dynamic molecular analysis of pretreatment tumor tissue demonstrated that not only the density of the immune cell infiltrate, but rather its functional properties correlated with the response to ICI therapy. In particular, the predominance of central memory $\mathrm{T}\left(\mathrm{T}_{\mathrm{CM}}\right)$ cells with a diverse T-cell receptor (TCR) repertoire were associated with a favorable treatment outcome. $^{5}$ On the other hand, we did not observe any predictive potential on previously suggested molecular biomarkers such as tumor PD-L1 expression or MCPyV status. ${ }^{256}$ Thus, with the present study we aimed at testing clinically well applicable predictive biomarkers in a larger patient cohort (114 MCC patients, although these included 41 patients from our earlier study ${ }^{5}$ by expanding our initial oligocentric approach to a multicentric study, but also limiting the complexity of the molecular analyses to those showing the highest predictive value in our previous study.

\section{MATERIALS AND METHODS Patients and samples}

One hundred and fourteen $(\mathrm{n}=114)$ patients treated between May 2018 and July 2020 at 11 MCC referral centers (Bochum, Buxtehude, Erlangen, Essen, Heidelberg, Homburg, Lübeck, Mainz, Regensburg, Stockholm, Tübingen) were retrospectively identified according to the following selection criteria: histopathologically confirmed diagnosis of MCC, treatment with PD-1/PD-L1 ICI for unresectable advanced disease, and complete follow-up documentation of ICI therapy outcome including best overall response (BOR), progression-free (PFS) and overall (OS) survival. BOR was determined according to RECIST V.1.1. ${ }^{7}$ PFS and OS were defined as time from therapy start until disease progression or death, respectively; if no such event occurred, the date of the last patient contact was used as endpoint of survival assessment (censored observation). Detailed clinical parameters at baseline of ICI therapy were collected from the patients' medical charts; it is important to note that a subgroup of 41 patients had already been described in an earlier study ${ }^{5}$ (online supplemental table S1). Immunosuppression was assigned to patients suffering from hematological neoplasia or to patients treated with multiple drugs for multiple cancers or immunosuppressive medications. If available, pretreatment samples of formalinfixed paraffin-embedded (FFPE) tumor tissue from the studied patients were collected for molecular analysis.

\section{Detection of MCPyV DNA}

Detection of MCPyV DNA was performed as previously described by TaqMan Real-Time qPCR using the following large T-antigen (LTA) specific primers and TaqMan probe: forward primer; CCA AAC CAA AGA ATA AAG CAC TGA; reverse primer, TCG CCA GCA TTG TAG TCT AAA AAC, and probe: FAM-AGC AAA AAC ACT CTC CCC ACG TCA GAC AG-BHQ1. ${ }^{5}$

\section{Multiplex immunofluorescence staining}

Multiplex immunofluorescence staining of FFPE tumor tissue was performed using the Opal chemistry (PerkinElmer, Waltham, USA, Cat.No.: OP7TL4001KT) with two panels of antibodies, ie, against CD4, CD8, CD20, Foxp3 and CD68 (panel 1), or CD27, GZMB, TCF1, CD45RA and CD45RO (panel 2). Synaptophysin served as tumor marker in either panels. Briefly, after deparaffinization and fixation, $3 \mu \mathrm{m}$ tumor sections were processed with retrieval buffers for $15 \mathrm{~min}$ in an inverter microwave oven. Thereafter, sections were incubated with the antibody diluent for $10 \mathrm{~min}$ at room temperature, followed by incubation with the primary antibody for $30 \mathrm{~min}$. After applying Opal polymer HRP secondary antibody and Opal fluorophore solution each for $10 \mathrm{~min}$, antibodies were removed by microwave treatment before a further round of staining. The antibodies, their dilutions, the respective retrieval buffers as well as the sequence of usage are described in detail in online supplemental table S2. Visualization of the different fluorophores was achieved on the Mantra Quantitative Pathology Imaging System (PerkinElmer). For each tumor sample, quantification of the different cell types was performed at medium magnification on three randomly selected areas located either in the juxtatumoral or intratumoral region in a semiautomatic fashion with the InForm Tissue Analysis software (Akoya Biosciences, Menlo Park, USA). Since tumor samples were received from different pathology institutes, the quality of the FFPE material was not uniform, resulting in variations in fluorescence intensity from sample to sample. To avoid quantification errors due to these intensity variations, the InForm tissue analysis software was trained on tumor tissue samples from the respective sources, thus developing an algorithm based on the median of the determined intensities. Subsequently, training of the software was performed on five different MCC tissue samples to recognize staining patterns/cell types. Finally, a principal components analysis was used to visualize possible pattern of the immunofluorescence staining results across the samples as suggested by Shen $e t$ $a l .^{8}$ Annotation by the different pathology institutes the samples were received from no significant batch effect was observed indicating the initial training of the InForm software was sufficient.

Two independent observer, blinded to ICI response, monitored the quantification analysis and classified the respective cell types in relation to all nucleated cells per sample into five categories: $0 \%,>1 \%, 1 \%-5 \%$, $5 \%-10 \%$ and $>10 \%$. Disagreements were resolved by 
taking the opinion of a third observer into consideration. Markers used for the quantification of the different immune cell types are listed in online supplemental table S3 and the raw data of the quantification analysis by InForm Tissue Analysis software is provided in online supplemental table S4 and S5 for panel 1 and panel 2, respectively.

\section{STATISTICAL ANALYSES}

A Bayesian cumulative ordinal regression model was applied for predicting PD-1/PD-L1 ICI therapy response in MCC patients. The model was fit with a dataset consisting of 114 patients and 15 clinical parameters; year of treatment and participation in a clinical trial were considered as possible confounders (table 1). Model parameters were described with probability distributions that take into account the uncertainty of the estimates. Treatment response was classified into $\mathrm{CR}$, partial response (PR), stable disease (SD) and progressive disease (PD) on an ordinal scale. We applied a cumulative ordinal regression model to the data, which takes the ordinal nature of the response variable into account. Compared with other approaches that incorrectly treat the response variable as metric (such as linear regression) or nominal (eg, by binarizing the response variable), this may lead to more precise inference and therefore reduces over- or underestimation of effect sizes. ${ }^{9}$ The cumulative regression model regards the tendency of a patient to respond to treatment as a latent (=unobserved) variable that is determined by the patient characteristics. The model is described by the following formula: ${ }_{i}=\beta_{\text {age. }} x_{i, \text { age }}+\beta_{L D H} \cdot x_{i, L D H} \ldots$; in which is the location of the latent variable for patient $i$, $\beta_{\text {age }}$ is the $\beta$ coefficient for the predictor age and $x_{i, \text { age }}$ is the indicator variable of patient $i$ for age. The $\beta$ coefficient of the predictor provides information on whether or not a predictor is associated with a higher probability of treatment response. Student $t$-priors with 7 degrees of freedom and a SD of 1 were chosen as weakly informative priors for the $\beta$ coefficients. Model fit is performed numerically by Markov chain Monte Carlo. ${ }^{10}$ The width of the distribution gives an impression of the uncertainty of the estimate: a distribution tightly concentrated around a value means that the dataset allows for a precise estimate of that parameter, while a broader distribution means that the data is consistent with a wide range of parameter values. Average predictive comparisons are calculated as expected changes in response associated with a unit difference in one of the inputs. They were calculated with respect to having at least a PR to treatment, for example, for immunosuppression, values between $-0 \%$ and $-40 \%$ denote that comparing a patient with immunosuppression to an otherwise identical patient without immunosuppression, the patient with immunosuppression has (on average) a $0 \%-40 \%$ lower probability of having at least a PR to treatment. Fitting the model to the dataset was done with the $\mathrm{R}$ software package 'brms', which utilizes 'Stan' in the background. ${ }^{11}$ Missing values were estimated by multiple imputation with the $\mathrm{R}$ package 'mice'. ${ }^{11-13}$ The imputed data values are consistent with the observed data (online supplemental figure 1). Using leaveone-out cross-validation (LOO), this model has a similar (expected log-predictive density (ELPD), a measure of its ability to generalize to unseen data) as a sequential model without category-specific effects, meaning that including category-specific effects does not improve model performance and the proportional odds assumption does not have a strong effect on the model conclusions. In the following, we report the detailed results for LOO and ELPD for completeness (online supplemental figure 2). A detailed description is given in online supplemental materials and methods. Transparent Reporting of a multivariable prediction model for Individual Prognosis or Diagnosis reporting guidelines were followed to develop the predictive model, including patient selection. ${ }^{14}$

Kaplan-Meier plots were generated with R V.3.5.1 using the package 'survival' (V.2.44-1.1 and survminer V.0.4.6). All patients with PFS and/or OS of more than 36 months are censored without having a respective event, because the data beyond this period is very sparse. Log rank test is used to calculate $\mathrm{p}$ values.

For statistical testing of T-cell abundance in MCC tumor tissue, $\mathrm{p}$ values were determined by beta regression with R V.4.0.2 and packages 'Imtest' and 'betareg' (V.0.9-38 and V.3.1-4).

For statistical testing of the distance between tumor cells and $\mathrm{CD}^{+} \mathrm{T}$ cells in tumor tissue, the unpaired twotailed Student's t-test, calculated in GraphPad Prism V.5 (San Diego, USA) was applied.

\section{RESULTS}

Patient characteristics, response to ICI and survival outcomes A total of 114 patients treated with PD-1/PD-L1 ICI (avelumab, $\mathrm{n}=57$; nivolumab, $\mathrm{n}=13$; pembrolizumab, $\mathrm{n}=44$ ) for unresectable advanced MCC were identified at 11 MCC referral centers in Germany and Sweden. Detailed patient characteristics are given in table 1. Of 114 patients, 54 (47\%) experienced an objective response (BOR=CR/PR), and $74 / 114$ patients $(65 \%)$ a disease control $(\mathrm{BOR}=\mathrm{CR} / \mathrm{PR} / \mathrm{SD})$ on ICI (figure $1 \mathrm{~A})$. When the entire patient cohort was divided by type of therapy, in the cohort treated with the anti-PD-L1 antibody avelumab, 22/57 patients (39\%) experienced an objective response and $33 / 57$ patients (58\%) disease control; in the cohort treated with the anti-PD-1 antibodies pembrolizumab or nivolumab, an objective response was observed in $32 / 57$ patients $(56 \%)$ and disease control in $41 / 57$ patients (72\%). Of 114 patients, $37(32 \%)$ died within a median follow-up time of $12.0( \pm 2.41,95 \%$ CI $)$ months. KaplanMeier estimates for PFS and OS categorized by BOR (CR, $\mathrm{n}=24 ; \mathrm{PR}, \mathrm{n}=30 ; \mathrm{SD}, \mathrm{n}=20$; and $\mathrm{PD}, \mathrm{n}=40$ ) revealed a clear separation of the curves for patients experiencing disease control $(\mathrm{CR} / \mathrm{PR} / \mathrm{SD})$ as BOR compared with those presenting a primary progression on therapy (online supplemental figure 3). The median PFS and OS in the 
Table 1 Patient and tumor characteristics at baseline of anti-PD-1/PD-L1 therapy

\begin{tabular}{lll} 
All patients $n=114(100 \%)$ & $\begin{array}{l}\text { Disease control (BOR=CR/PR/ } \\
\text { SD) } n=74(100 \%)\end{array}$ & $\begin{array}{l}\text { Disease progression (BOR=PD) } \\
n=40(100 \%)\end{array}$ \\
\hline
\end{tabular}

Patient characteristics

Gender

\begin{tabular}{|c|c|c|c|}
\hline Male & $82(72 \%)$ & $54(73 \%)$ & $28(70 \%)$ \\
\hline Female & $32(28 \%)$ & $20(27 \%)$ & $12(30 \%)$ \\
\hline \multicolumn{4}{|l|}{ Age } \\
\hline$\geq 70$ & $74(65 \%)$ & $50(68 \%)$ & $24(60 \%)$ \\
\hline \multicolumn{4}{|c|}{ Overall performance status (ECOG) } \\
\hline$\geq 1$ & $49(43 \%)$ & $26(35 \%)$ & $23(57)$ \\
\hline Not available & $1(1 \%)$ & $1(1 \%)$ & $0(0 \%)$ \\
\hline \multicolumn{4}{|l|}{ Immunosuppression } \\
\hline No & $92(81 \%)$ & $64(86 \%)$ & $28(70 \%)$ \\
\hline Yes & $22(19 \%)$ & $10(14 \%)$ & $12(30 \%)$ \\
\hline Not available & $4(3 \%)$ & $3(4 \%)$ & $1(2 \%)$ \\
\hline \multicolumn{4}{|l|}{ CRP (blood) } \\
\hline Normal & $30(26 \%)$ & $21(28 \%)$ & $9(23 \%)$ \\
\hline Elevated & $55(48 \%)$ & $31(42 \%)$ & $24(60 \%)$ \\
\hline Not available & $29(26 \%)$ & $22(30 \%)$ & $7(16 \%)$ \\
\hline \multicolumn{4}{|l|}{ NLR (blood) } \\
\hline$<4$ & $54(47 \%)$ & $36(49 \%)$ & $18(45 \%)$ \\
\hline$\geq 4$ & $35(31 \%)$ & $20(27 \%)$ & $15(38 \%)$ \\
\hline Unknown primary & $15(13 \%)$ & $9(12 \%)$ & $6(15 \%)$ \\
\hline \multicolumn{4}{|c|}{ Metastatic stage (AJCC) } \\
\hline MO & 17 (15\%) & $11(15 \%)$ & $6(15 \%)$ \\
\hline M1a & $36(32 \%)$ & $23(31 \%)$ & $13(32 \%)$ \\
\hline M1b/M1c & $61(53 \%)$ & $40(54 \%)$ & $21(53 \%)$ \\
\hline \multicolumn{4}{|l|}{ Organs involved } \\
\hline 1 & $51(45 \%)$ & $38(51 \%)$ & $13(32 \%)$ \\
\hline$>1$ & $63(55 \%)$ & $36(49 \%)$ & $27(68 \%)$ \\
\hline \multicolumn{4}{|l|}{ MCPyV status (tumor) } \\
\hline Negative & $10(9 \%)$ & $6(8 \%)$ & $4(10 \%)$ \\
\hline Positive & $32(28 \%)$ & $20(27 \%)$ & $12(30 \%)$ \\
\hline Not available & 72 (63\%) & $48(65 \%)$ & $24(60 \%)$ \\
\hline \multicolumn{4}{|l|}{ PD-L1 (tumor) } \\
\hline Negative & 17 (15\%) & $11(15 \%)$ & $6(15 \%)$ \\
\hline
\end{tabular}

Continued 
Table 1 Continued

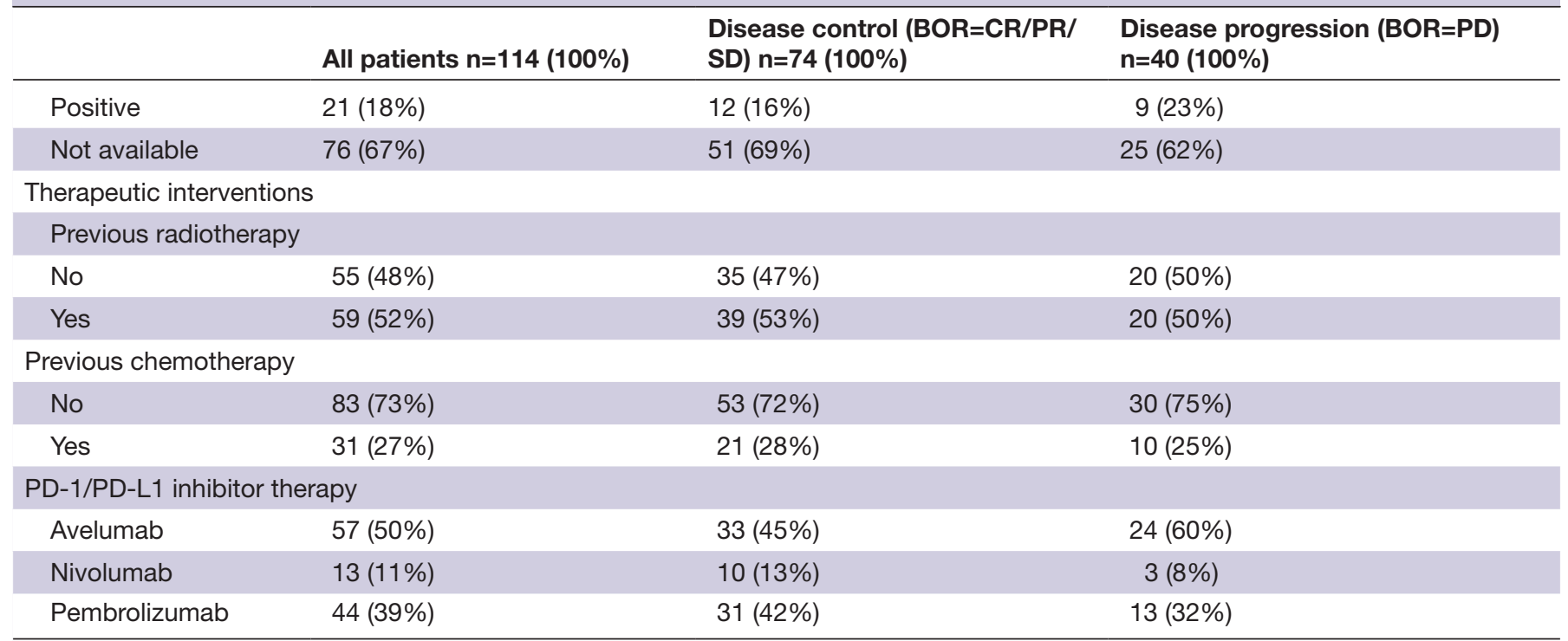

AJCC, American Joint Committee on Cancer; BOR, best overall response; CRP, C reactive protein; ECOG, Eastern Cooperative Oncology Group; LDH, lactate dehydrogenase; MCPyV, Merkel cell polyomavirus; NLR, neutrophil to lymphocyte ratio.

control group were 12.1 and 15.9 months, and 1.4 and 3.9 months, respectively, in the progression group.

On the finding of this clear separation in survival probabilities between patients responding with disease control $(\mathrm{BOR}=\mathrm{CR} / \mathrm{PR} / \mathrm{SD})$ and patients responding with disease progression $(\mathrm{BOR}=\mathrm{PD})$, we performed all further molecular analyses on the association of clinical and molecular characteristics with therapy response based on the discrimination between these two patient groups (ie, disease control group vs disease progression group). Due to the limited number of samples, we refrained from forming further subclusters taking the degree of response into account.

\section{Baseline clinical parameters are associated with a favorable response to ICI therapy}

Most predictive models dichotomize response to therapy, which neglects the extent of the response. To overcome this limitation, we developed a Bayesian model that instead of dichotomizing the therapy response into two groups, that is, regression versus progression, rather reflects the established clinical response evaluation

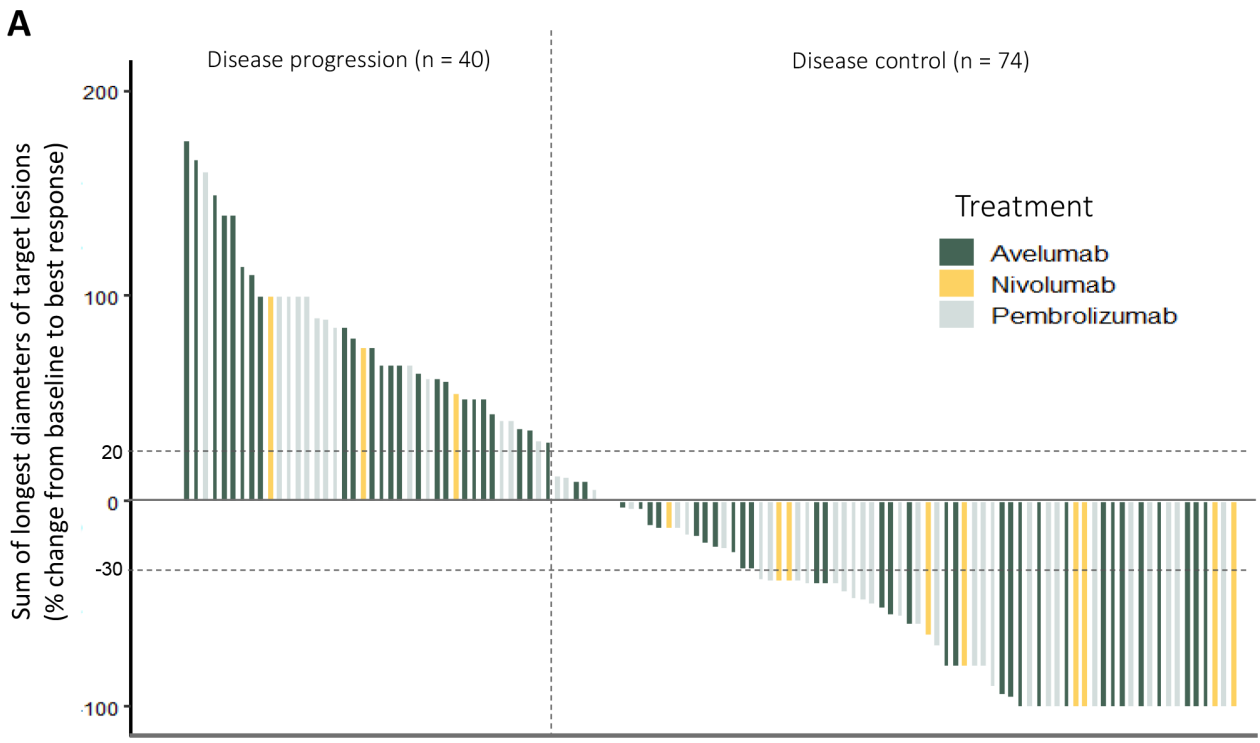

Figure 1 Response of $n=114$ advanced MCC patients on PD-1/PD-L1 immune checkpoint inhibition therapy. Waterfall plot depicting the best overall response (BOR) as change in the sum of the longest diameters of target lesions from baseline to BOR. Each bar, color coded by therapeutic antibody, represents an individual patient. The pointed vertical line discriminates patients with disease control (BOR=CR/PR/SD) from patients with disease progression (BOR=PD). CR, complete response; MCC, Merkel cell carcinoma; PD, progressive disease; PR, partial response; SD, stable disease. 
criteria in solid tumors (RECIST): CR, PR, SD, and PD. ${ }^{7}$ Resulting from this Bayesian model, we found the absence of immunosuppression as well as a limited number of organs (1 vs $>1$ ) involved into disease spread as the strongest predictors of a favorable response to PD-1/PD-L1 ICI therapy (figure 2). Interestingly, the involved organ type, for example, soft tissue versus visceral, showed less predictive power. These calculations indicate that immunocompetent patients or patients with only one affected organ have probability to achieve disease control on ICI treatment, that is by about $20 \%$ higher $(0 \%-40 \%$ increase contains almost all the probability). Additionally, an unimpaired overall performance status (Eastern Cooperative Oncology Group $(\mathrm{ECOG})=0$ ), patient age of 70 years and above, as well as normal lactate dehydrogenase $(\mathrm{LDH})$ and $\mathrm{C}$ reactive protein (CRP) serum levels were associated with a higher probability of disease control on ICI, but to a lower extent. Interestingly, patients' sex, localization of the primary, pretreatment with radiation or chemotherapy, and MCPyV status or PD-L1 expression of the tumor revealed no relevant association with ICI therapy response. Similarly, when we tested if year of treatment and participation in a clinical trial were possible confounders, no impact on the therapeutic outcome was observed. Surprisingly, in our investigated patient cohort with an equal distribution of PD-L1 and PD-1 ICI therapies (see table 1), the use of anti-PD-1 antibodies for ICI therapy was associated with a higher probability of a favorable therapy response (figure 2). Notably, the distribution of relevant patient and tumor characteristics, particularly immunosuppressive state, number of organs involved with disease, impaired ECOG status, and elevated serum LDH and CRP were equally distributed among the two treatment cohorts, that is, anti-PD-1 and anti-PD-L1 antibody ICI (online supplemental table S6).

\section{Pretreatment dense intratumoral infiltrates of $\mathrm{CD}^{+} \mathrm{T}_{\mathrm{CM}}$ are associated with a favorable response to ICI}

We recently demonstrated by transcriptomics, spatial proteomics and TCR sequencing of sequential tumor biopsies under PD-1/PD-L1 ICI therapy that a predominance of $\mathrm{T}_{C M}$ with a diverse TCR repertoire and the ability to expand on ICI is associated with a favorable therapy response. ${ }^{5}$ This approach allows a good understanding of the complex immune biology of MCC, but is difficult to use in the clinical routine of patient care. In order to establish clinically well applicable predictive biomarkers, we here limited the complexity of our molecular investigations to multiplexed immunohistochemistry of pretreatment FFPE tumor tissue in order to extract the most important cell type characteristics for therapy response, which can be realistically analyzed as predictive biomarker in the future. Phenotyping of the immune infiltrate of pretreatment tumor tissue samples of 21 patients, including some tumors from our earlier report, ${ }^{5}$ (11 patient with disease control, 10 patients with disease progression) for the expression of CD4, CD8, CD20, Foxp3, and CD68 showed that dense immune cell infiltration, particularly by $\mathrm{CD} 8^{+} \mathrm{T}$ cells, correlated with a favorable ICI therapy response (figure 3A,B). Significantly more $\mathrm{CD} 8^{+} \mathrm{T}$ cells were infiltrating the juxtatumoral area $(p=0.02)$ and higher number of cytotoxic $\mathrm{T}$ cells were present in the intratumoral area $(\mathrm{p}=0.16)$ of patients achieving disease control (figure 3C, online supplemental figure 4). Figure 3D illustrates how the spatial distribution of the tumor-infiltrating $\mathrm{CD} 8^{+} \mathrm{T}$ cells was measured as the distance between the nuclei of $\mathrm{CD}^{+} \mathrm{T}$ cells and synaptophysin $^{+}$MCC cells. For distance analysis, tumor samples with $\mathrm{CD}^{+} \mathrm{T}$ cells that were less than $1 \%$ of total cells had to be excluded, because in these cases it was not possible to measure the distance of at least 20 different tumor/Tcell pairs. In patients with disease control, $\mathrm{CD} 8^{+} \mathrm{T}$ cells were in direct and close contact with the tumor cells with a mean distance length of $13.24 \mu \mathrm{m}$, whereas the mean distance length was significantly higher in patients with disease progression $(22.00 \mu \mathrm{m}, \mathrm{p}=0.009)$ (figure $3 \mathrm{E}$ ). Moreover, in patients showing disease progression, the $\mathrm{CD}^{+} \mathrm{T}$ cells were mostly restricted to the juxtatumoral stromal space, and only rarely within the tumor tissue. We did not detect significant differences in the amount or distribution of regulatory $\mathrm{T}$ cells $\left(\mathrm{CD}^{+} \mathrm{FoxP}^{+}\right)$, B cells $\left(\mathrm{CD} 20^{+}\right)$, and monocytes/macrophages $\left(\mathrm{CD} 68^{+}\right)$between tumor tissues of patients with disease control and patients with disease progression. However, with respect to $\mathrm{CD}^{+} 0^{+} \mathrm{B}$ cells within the cellular immune infiltrate, it is important to note that their frequency varied strongly between samples.

Staining for CD27, GZMB, TCF1, CD45RA and CD45RO allows a precise distinction of $\mathrm{T}_{\mathrm{CM}}$ and effector $\mathrm{T}$ cells with $\mathrm{T}_{\mathrm{CM}}$ characterized by colocalization of CD27, TCF1 and CD45RO. Indeed, only in pretreatment tumors from patients with disease control, we observed a clear co-localization of these $\mathrm{T}_{\mathrm{CM}}$ markers (figure 4). Moreover, quantification of T-cell subtypes confirmed a higher percentage of $T_{C M}$ of total tumor infiltrating lymphocytes (TILs) number in the intratumoral infiltrate as well as in the juxtatumoral area (table 2). Effector T cells characterized by colocalization of GZMB and CD45RA were also more frequently observed in the cellular tumor infiltrate of pretreatment tumor tissue of patients with disease control than in those with disease progression; however, the difference was less evident ( $\mathrm{p}=0.07$; online supplemental figure 5). It should be noted that CD45RA re-expression has also been described in terminally differentiated $\mathrm{T}$ cells characterized by decreased proliferative capacity, increased senescence signaling in vitro. ${ }^{15}$

\section{DISCUSSION}

To find predictors, which assess the individual probability of success of PD-1/PD-L1 ICI in MCC, we collected clinical information on 114 accordingly treated patients and established the spatial distribution of tumor infiltrating $\mathrm{T}$ cells as well as their activation and differentiation status in pretreatment FFPE tumor tissue samples by two IHC panels. We developed a Bayesian cumulative ordinal 


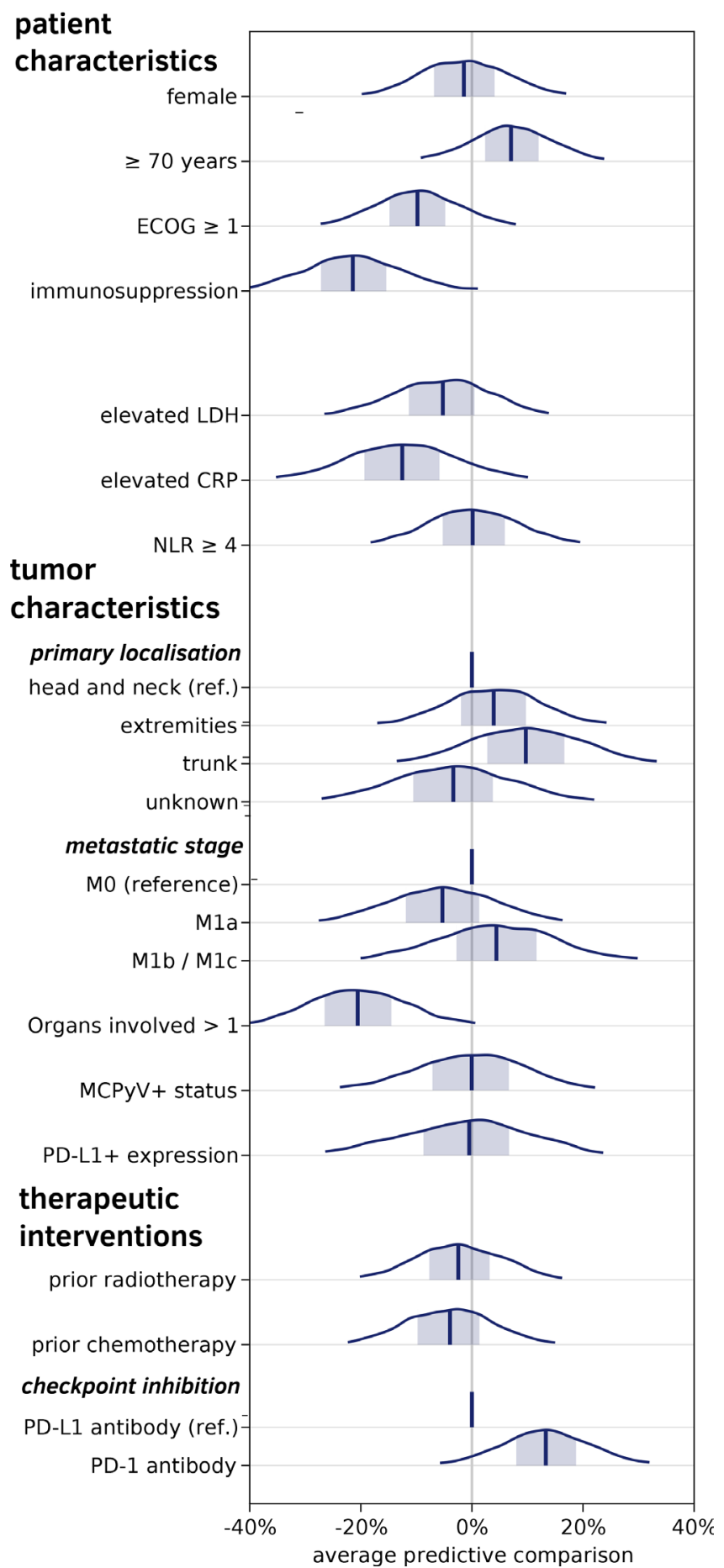

Figure 2 Best overall response (BOR) to anti-PD-1/PD-L1 therapy in correlation to baseline clinical patient and tumor characteristics. The correlations are visualized by average predictive comparisons calculated by a Bayesian cumulative ordinal regression model. While the presented data refer to the full model using four categories of response: $C R, P R, S D$, and $P D$, to ease interpretation we mapped the obtained results by average predictive comparisons on a single probability scale for disease control $(\mathrm{BOR}=\mathrm{CR} / \mathrm{PR} / \mathrm{SD})$ and disease progression $(\mathrm{BOR}=\mathrm{PD})$ as a probability distribution, given as the percentage of average predictive comparison. The $95 \%$ credibility intervals are colored in light blue. Distinct parameters are marked as reference (Ref), described as vertical blue lines set at $0 \%$ average predictive comparison. CR, complete response; CRP, $\mathrm{C}$ reactive protein; ECOG, Eastern Cooperative Oncology Group; LDH, lactate dehydrogenase; MCPyV, Merkel cell polyomavirus; NLR, neutrophil to lymphocyte ratio; PD, progressive disease; PR, partial response; SD, stable disease. 
A
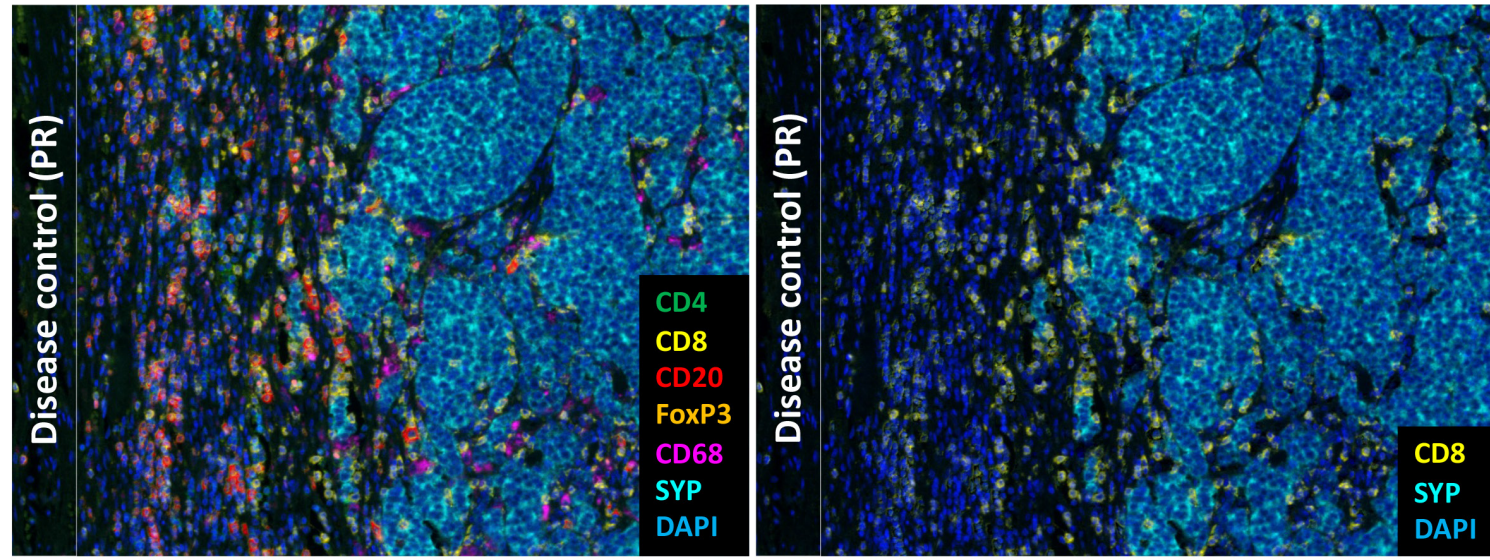

B
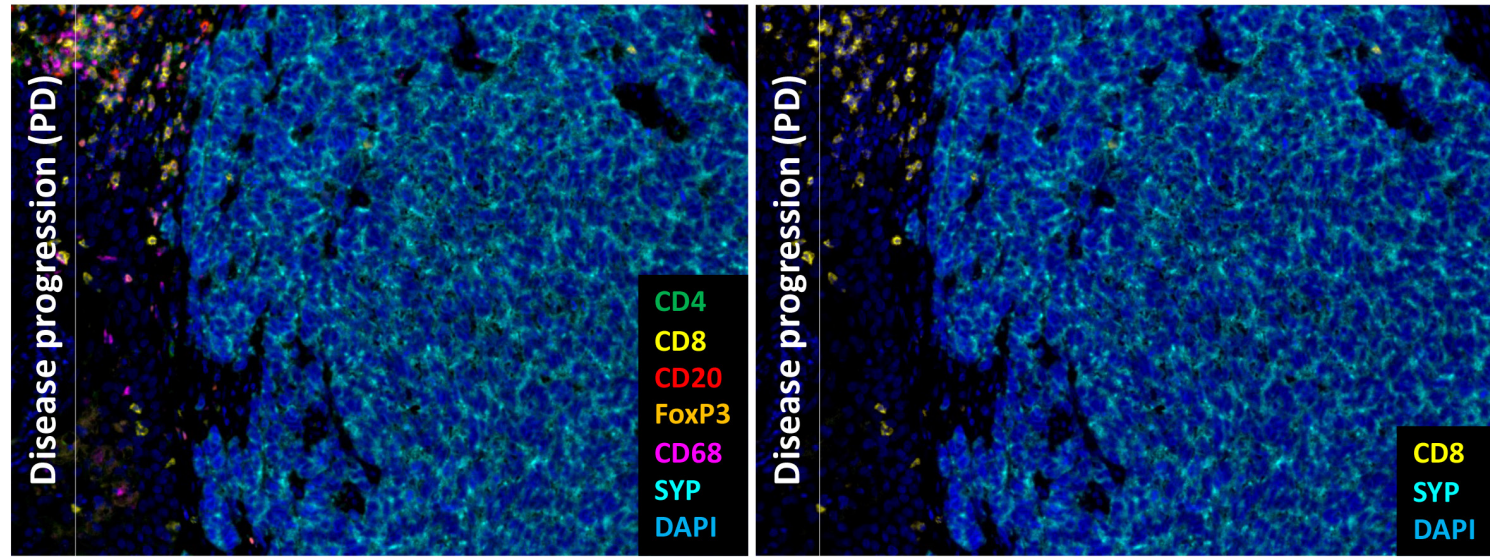

C
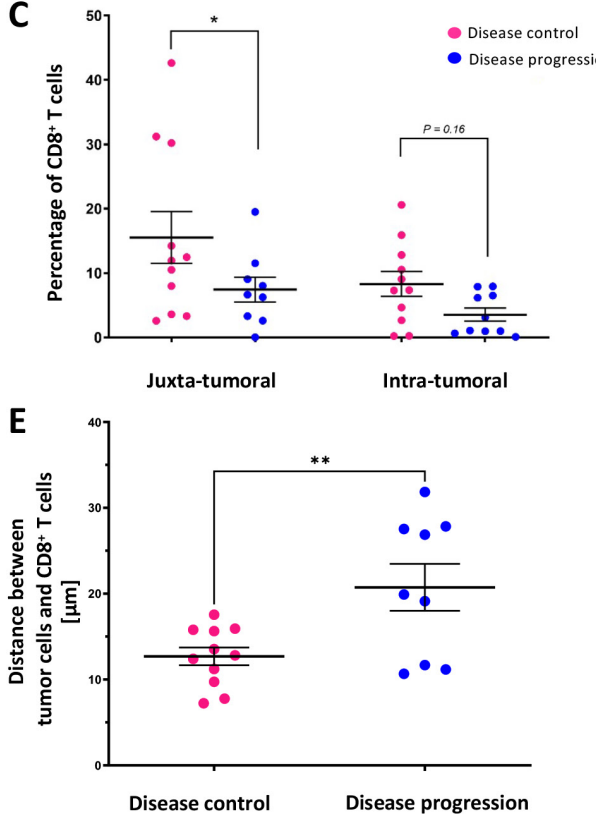

D

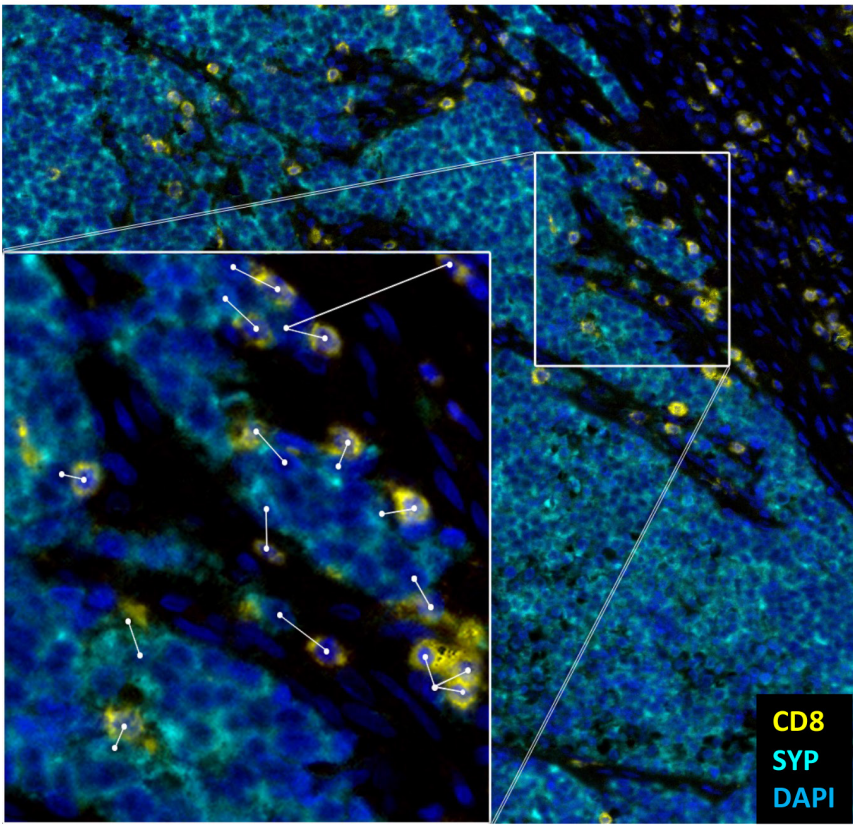

Figure 3 High density of tumor-infiltrating $C D 8^{+}$central memory T cells in close proximity to tumor cells in MCC patients showing disease control (CR/PR/SD) on PD-1/PD-L1 ICl phenotyping of the cellular immune infiltrate present in MCC tumor lesions obtained at baseline of $\mathrm{ICl}$ therapy of a representative patient responding with disease control $(\mathrm{A})$ and disease progression (B) was done by multiplexed immunohistochemistry-based staining using antibodies against CD4 (green), CD8 (yellow), CD20 (red), FOXP3 (orange), CD68 (magenta), and the MCC marker synaptophysin (SYN) (cyan); nuclei are stained with DAPI (blue). depicted are merged images at $\times 20$ magnification. (C) Percentage of CD8 ${ }^{+} \mathrm{T}$ cells in pretreatment tumor tissue from patients showing disease control and those showing disease progression in the juxtatumoral and intratumoral area. $\mathrm{P}$ values were determined using beta regression. (D) Measurement of the distance between CD8 ${ }^{+} \mathrm{T}$ cells and tumor cells. (E) Mean value of the distance between $\mathrm{CD} 8^{+} \mathrm{T}$ cells and tumor cells for patients showing disease control and those showing disease progression. P values were determined using unpaired, two-tailed Student's t-test. CR, complete response; ICl, immune checkpoint inhibitor; MCC, Merkel cell carcinoma; PR, partial response; SD, stable disease. 

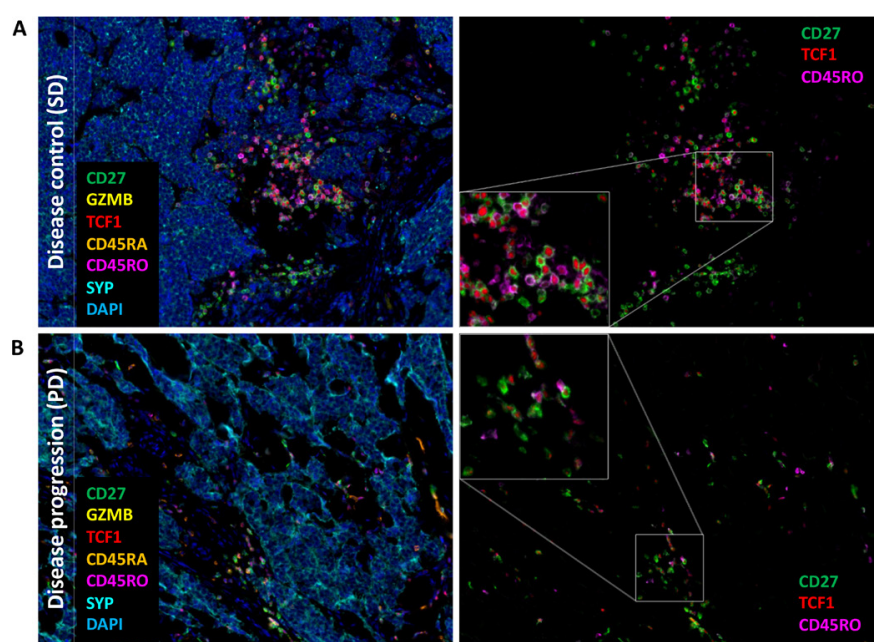

Figure 4 Predominance of central memory $T$ cells $\left(T_{C M}\right)$ among tumor-infiltrating lymphocytes of patients showing disease control (CR/PR/SD) on PD-1/PD-L1 ICI therapy. Multiplexed immunofluorescence staining of pretreatment tumor tissue from a representative patient showing disease control $(A)$ and disease progression (B) using antibodies against CD27 (green), GZMB (yellow), TCF1 (red), CD45RA (orange), CD45RO (magenta), and the MCC marker synaptophysin (SYN) (cyan); nuclei are stained with DAPI (blue). Depicted are merged images at $\times 20$ magnification. To visualize the colocalization of CD27, TCF1 and CD45RO, an enlarged image view is shown. CR, complete response; $\mathrm{ICl}$, immune checkpoint inhibitor; MCC, Merkel cell carcinoma; $\mathrm{PR}$, partial response; SD, stable disease.

regression model that includes the distance between clinical characteristics and thereby appropriately accounts for category order. This model avoids problems such as dichotomizing the outcome or treating the distance between the categories as equal, and thus uses the available data efficiently. It revealed the absence of immunosuppression and the metastatic involvement of a limited number of organ systems as characteristics predicting disease control on PD-1/PD-L1 ICI therapy with the highest probability. Additional characteristics associated with treatment response were age, overall performance status, serum LDH and serum CRP, as well as a brisk intratumoral infiltrate by $\mathrm{T}_{\mathrm{CM}}$ (figure 5). However, both the data model and the missing data model rely on assumptions about the data generating process, for example, that data are missing at random. Even if the implications of these assumptions have been evaluated carefully, all results are still conditioned on the underlying model and should be interpreted with this in mind.

The expanded patient cohort under investigation of $n=114$ that allowed fitting a more complex Bayesian model supported our previously published observations in 41 patients. ${ }^{5}$ The positive effect of an intact immune system observed in either study was also reported from the avelumab expanded access program for metastatic MCC patients in which immunocompromised patients achieved a lower response rate with shorter durations of response. ${ }^{16}$ It should be noted that other characteristics associated with a lower probability of response, such as a limited performance status $($ ECOG $>0$ ) or an elevated serum CRP, are also likely to reflect an impaired immune status of the respective patient. Similarly, an elevated serum LDH level and a higher number of involved organs are not to be interpreted only as markers of a higher tumor load. Notably, in our previous report, we dichotomized the number of involved organs up to two or more, these groups did not show a clear association with the probability of response but had broad posterior intervals; in contrast, the larger cohort is consistent with the involvement of only one organ being a strong positive predictive marker. Patient age of 70 years and above was also found to be associated with a higher probability of disease control, but to a lesser extent. A positive correlation of response to anti-PD-1 therapy and patient age $\geq 60$ years was described in melanoma. ${ }^{17}$ In this respect, it is important to note that chronological age does not necessarily reflect immunological age. One factor that correlates better with biological/immunological age than chronological age is frailty, which directly describes a person's health status. ${ }^{18}$ For example, the process of inflammation is a predictor for frailty and one of the key cell types believed to facilitate an inflammatory phenotype are tumor-infiltrating macrophages, which are often detected in MCC tissue. ${ }^{19}$ Other clinical characteristics such as sex, primary site, prior radiation or chemotherapy, PD-L1 expression by tumor cells, and tumor MCPyV status did not show relevant association to PD-1/PD-L1 ICI response. These observations are in line with the results from a study scrutinizing $37 \mathrm{MCC}$ patients receiving ICI. ${ }^{20}$

Functional characterization of the immunological infiltrate of pretreatment tumor tissue revealed that in particular the presence of $\mathrm{T}_{\mathrm{CM}}$ in close proximity to tumor cells was associated with a favorable response to ICI therapy. It is important to note that because the tumor samples were received from different pathology institutes, the quality of the FFPE material was not uniform, resulting in variations in fluorescence intensity from sample to sample. To avoid quantification errors due to these intensity variations, the InForm tissue analysis software was trained on tumor tissue samples from the respective sources, thus developing an algorithm based on the median of the determined intensities. Moreover, the observation was consistent with our previous work, where we performed transcriptomics, spatial proteomics and TCR sequencing of sequential tumor biopsies before and under ICI therapy of rather uniform quality. These observations confirm the robustness of the chosen approach and the importance of $\mathrm{T}_{\mathrm{CM}}$ as one of the effectors of response to ICI therapy. The superior antitumor efficacy of $\mathrm{T}_{\mathrm{CM}}$ cells can be explained by their low activation threshold, rapid proliferative and differentiation capacity on cognate activation, as well as their capacity for long-term persistence facilitating immunologic memory. ${ }^{2122}$ Indeed, since $\mathrm{T}_{\mathrm{CM}}$ cells are the major source of secondary effector cells during a recall response, the duration of anti-tumor immune responses depends 
Table 2 Quantification of the cellular tumor infiltrate characterized by multiplex immunohistochemistry staining

Pretreatment MCC tumor tissue samples

\begin{tabular}{|c|c|c|c|c|c|}
\hline \multirow{2}{*}{ Response to CPI } & & \multicolumn{2}{|c|}{ Total leucocyte no per observed area } & \multicolumn{2}{|c|}{$\begin{array}{l}T_{C M} \text { in \% of total lymphocyte no per } \\
\text { observed area }\end{array}$} \\
\hline & & Juxtatumoral & Intratumoral & Juxtatumoral & Intratumoral \\
\hline \multirow{12}{*}{ Disease control } & PR & 2121 & 731 & 4.7 & 2.2 \\
\hline & $\mathrm{CR}$ & 1510 & 989 & 3.0 & 2.7 \\
\hline & $\mathrm{CR}$ & 1813 & 3519 & 0.0 & 22.0 \\
\hline & SD & 5436 & 9405 & 18.0 & 11.7 \\
\hline & PR & 1902 & 2162 & 6.7 & 8.0 \\
\hline & PR & 1451 & 97 & 13.0 & 26.7 \\
\hline & SD & 115 & 283 & 23.0 & 33.0 \\
\hline & $\mathrm{CR}$ & 1 & 14 & 0.0 & 0.0 \\
\hline & PR & 1846 & 6030 & 0.0 & 11.0 \\
\hline & PR & 1681 & 2080 & 13.0 & 11.7 \\
\hline & $\mathrm{CR}$ & 2261 & 428 & 33.0 & 36.7 \\
\hline & Mean value & 1831 & 2340 & 10.4 & 15.1 \\
\hline \multirow{11}{*}{$\begin{array}{l}\text { Disease } \\
\text { progression }\end{array}$} & $\mathrm{PD}$ & $N A$ & 0 & $N A$ & 0.0 \\
\hline & PD & 44 & 54 & 0.0 & 0.0 \\
\hline & PD & 1125 & 124 & 0.0 & 0.0 \\
\hline & PD & 239 & 170 & 12.0 & 15.0 \\
\hline & PD & 3831 & 2478 & 22.0 & 13.0 \\
\hline & PD & 316 & 376 & 17.0 & 10.0 \\
\hline & PD & 0 & 13 & 0.0 & 0.0 \\
\hline & PD & 2438 & 287 & 3.7 & 0.5 \\
\hline & PD & 403 & 62 & 2.0 & 1.7 \\
\hline & $\mathrm{PD}$ & 159 & 66 & 0.0 & 0.0 \\
\hline & Mean value & 950 & 363 & 6.3 & 4.0 \\
\hline
\end{tabular}

Tumor tissue samples were obtained from MCC patients prior to the start of PD-1/PD-L1 immune checkpoint inhibitor therapy. Lymphocytes were identified based on $\mathrm{CD}_{4} \mathrm{RA} \mathrm{A}^{+}$or $\mathrm{CD}_{4} 5 \mathrm{RO}^{+}$staining and the sum of both signals were used for the quantification of the total lymphocyte number per sample per observed area. $\mathrm{T}_{\mathrm{CM}}$ were determined based on the triple $\mathrm{CD} 27^{+} \mathrm{TCF} 1^{+} \mathrm{CD} 45 \mathrm{RO} \mathrm{O}^{+}$staining.

CPI, checkpoint inhibition; CR, complete response; MCC, Merkel cell carcinoma; PD, progressive disease; PR, partial response; SD, stable disease; $T_{C M}$, central memory T-cells.

on their presence. ${ }^{23}$ This is consistent with our present and recent findings as well as with further studies showing $\mathrm{T}_{\mathrm{CM}}$ characteristics to be effectively reactivated. ${ }^{24-27}$ Toews

et al demonstrated that $\mathrm{T}_{\mathrm{CM}}$-derived CAR $\mathrm{T}$ cells showed an augmented antitumor immunity against neuroblastoma cells under PD-1 blockade and subsequently formed

\begin{tabular}{|c|c|c|c|}
\hline & Patient characteristics & Tumor characteristics & Blood characteristics \\
\hline $\begin{array}{l}\text { Favors disease control } \\
\text { upon PD-1/PD-L1 } \\
\text { checkpoint inhibition therapy }\end{array}$ & $\begin{array}{l}\text { No immunosuppression } \\
\text { Age } \geq 70 \text { years } \\
\text { ECOG }=0\end{array}$ & $\begin{array}{l}\text { Only } 1 \text { organ involved } \\
\text { Brisk infiltrate of } \\
\text { effector/memory T cells }\end{array}$ & $\begin{array}{l}\text { Normal LDH } \\
\text { Normal CRP }\end{array}$ \\
\hline $\begin{array}{l}\text { Irrelevant for outcome } \\
\text { of PD-1/PD-L1 } \\
\text { checkpoint inhibition therapy }\end{array}$ & $\begin{array}{l}\text { Sex } \\
\text { Prior chemotherapy } \\
\text { Prior radiotherapy }\end{array}$ & $\begin{array}{l}\text { M stage }(\mathrm{M} 1 \mathrm{a} / \mathrm{b} / \mathrm{c}) \\
\text { MCPyVirus status } \\
\text { PD-L1 status }\end{array}$ & $\begin{array}{l}\text { Neutrophil to } \\
\text { Lymphocyte ratio }\end{array}$ \\
\hline $\begin{array}{l}\text { Favors disease progression } \\
\text { upon PD-1/PD-L1 } \\
\text { checkpoint inhibition therapy }\end{array}$ & $\begin{array}{l}\text { Immunosuppression } \\
\text { Age }<70 \text { years } \\
\text { ECOG } \geq 1\end{array}$ & $\begin{array}{l}\geq 2 \text { organs involved } \\
\text { No T-cell infiltrate }\end{array}$ & $\begin{array}{l}\text { Elevated LDH } \\
\text { Elevated CRP }\end{array}$ \\
\hline
\end{tabular}

Figure 5 Schematic overview on relevant clinical and molecular parameters determined before treatment and their predictive value on PD-1/PD-L1 ICI therapy response. CRP, C reactive protein; ECOG, Eastern Cooperative Oncology Group; ICI, immune checkpoint inhibitor; LDH, lactate dehydrogenase. 
a resident memory T-cell subset following tumor challenge. ${ }^{28}$ In non-small cell lung cancer patients treated with nivolumab, a longer PFS was observed in patients with a high $\mathrm{T}_{\mathrm{CM}} / \mathrm{T}_{\mathrm{EFF}}$-cell ratio in the circulation, suggesting an enrichment of peripheral circulating $\mathrm{T}_{\mathrm{CM}}$ subpopulations also as a potential positive predictive marker. ${ }^{29}$ Similarly, in hepatocellular carcinoma patients, according to midterm clinical trial results, an extended median relapse-free survival was associated with an increased $\mathrm{T}_{\mathrm{CM}^{-}}{ }^{-}$ subpopulation. ${ }^{22}$ Moreover, Siddiqui et al reported the presence of a $\mathrm{TCF} 1^{+} \mathrm{PD}-1^{+} \mathrm{CD} 8^{+}$T-cell subpopulation in the circulation of melanoma patients and among TILs of primary melanomas. In conclusion, the success of PD-1/ PD-L1 ICI therapy seems not to depend on the rejuvenation of differentiated exhausted $\mathrm{T}$ cells, but rather on the proliferation of the less-differentiated memory-like $\mathrm{CD} 8^{+}$ T cells. ${ }^{30}$

Long-lived memory T-cell formation and maintenance are driven by transcription factors like FOXO1, EOMES and TCF1. In particular, TCF1 was identified as the master regulator of genes, inducing serial T-cell reactivation and self-renewal. With respect to the limited predictive value of the presence of granzyme-expressing $\mathrm{T}$ cells in the tumor infiltrate, recently a granzyme-positive subpopulation of $\mathrm{CD}^{+} \mathrm{T}$ cells associated with age-related dysfunction of the immune system has been described. ${ }^{31}$ These cells are characterized by a pronounced tissue-homing capacity and a high clonality, that is, expressing only a limited diversity of TCRs, which might be of particular relevance for MCC, since this tumor affects mainly the elderly population. Indeed, we have shown in previous studies that high clonality of TIL in MCC is both a negative prognostic and predictive biomarker. ${ }^{532}$

In conclusion, our results provide a number of clinically well applicable baseline biomarkers associated with PD-1/PD-L1 ICI therapy response in patients suffering from advanced MCC. On a functional level, we confirmed the predominance of $\mathrm{T}_{\mathrm{CM}}$ among TILs in patients with a favorable ICI therapy response; a factor which can be determined on FFPE tissue.

\footnotetext{
Author affiliations

${ }^{1}$ Translational Skin Cancer Research, Deutsches Konsortium für Translationale Krebsforschung, Essen, Germany

${ }^{2}$ Department of Dermatology, University Hospital of Essen, Essen, Germany ${ }^{3}$ Department of Dermatology, University Hospital of Lübeck, Lübeck, Germany ${ }^{4}$ Department of Oncology-Pathology, Karolinska University Hospital, Stockholm, Sweden

${ }^{5}$ Department of Dermatology, University Hospital of Tübingen, Tübingen, Germany ${ }^{6}$ Department of Dermatology, University Medical Center Mainz, Mainz, Germany ${ }^{7}$ Department of Dermatology, University Hospital of Heidelberg, Heidelberg, Germany

${ }^{8}$ Deutsches Krebsforschungszentrum, Heidelberg, Germany

${ }^{9}$ Department of Dermatology, Ruhr University Bochum, Bochum, Germany

${ }^{10}$ Department of Dermatology, University Hospital Regensburg, Regensburg, Germany

${ }^{11}$ Department of Dermatology, Elbe Kliniken Buxtehude, Buxtehude, Germany

${ }^{12}$ Department of Dermatology, Saarland University Medical Center, Homburg, Germany

${ }^{13}$ Department of Dermatology-Oncology, University Hospital München, München, Germany
}

${ }^{14}$ Department of Dermatology, Skin Cancer Center Minden, Minden, Germany ${ }^{15}$ Department of Dermatology, Venereology and Allergology, University Medical Center Mannheim, Mannheim, Germany

${ }^{16}$ Deutsches Konsortium fur Translationale Krebsforschung, Essen, Germany

${ }^{17}$ Department of Pathology, University Hospital Essen, Essen, Germany

${ }^{18}$ Bioinformatics and Computational Biophysics, University of Duisburg-Essen, Duisburg, Germany

Acknowledgements We thank the patients who participated in this study, their families, and the staff members at the clinical centers who cared for them. We like to express our appreciation to Nalini Srinivas for critically proof reading the final version of the manuscript.

Contributors Conceptualisation: IS, SU, LK, H-US, DHa, DHo, and JCB; data curation: IS, SU, and JCB; formal analysis: IS, KH, and DHa; funding acquisition: JCB; investigation: IS, SU, LK, KH, DHa, DHo, and JCB; methodology: IS, SU, LK, DHa, DHo, and JCB; project administration: IS, SU, and JCB; resources: SU, LZ, PT, AM, HBA, LV, UL, TE, CL, JCH, TG, SH, PM, CP, LH, RG, JSU, DS, and JCB; software: IS, $\mathrm{KH}$, and DHa; supervision: SU, and JCB; validation: IS, SU, KH, DHa, DHo, and JCB; visualisation: IS, SU, KH, DHa, DHo, and JCB; writing-original draft: IS, SU, and JCB; writing-review and editing: IS, SU, LK, PT, LV, TE, JCH, TG, RG, KH, DHa, DHo, H-US, $\mathrm{DS}$, and JCB; IS, SU and JCB are guarantors of this study.

Funding This study was funded by the DKTK ED003

Competing interests SU declares research support from Bristol Myers Squibb and Merck Serono; speakers and advisory board honoraria from Bristol Myers Squibb,

Merck Sharp \& Dohme, Merck Serono, Novartis and Roche, and travel support from Bristol Myers Squibb, and Merck Sharp \& Dohme. LZ served as consultant and/or has received honoraria from Roche, Bristol-Myers Squibb, Merck Sharp \& Dohme, Novartis, Pierre-Fabre, and Sanofi; Research funding to institution: Novartis; travel support from Merck Sharp \& Dohme, Bristol-Myers Squibb, Amgen, Pierre-Fabre, and Novartis, outside the submitted work. PT declares invited speaker's honoraria from Bristol-Myers Squibb, Novartis, MSD, Pierre-Fabre, CureVac, Roche, Kyowa Kirin, Biofrontera, advisory board honoraria from Bristol-Myers Squibb, Novartis, Pierre-Fabre, Merck Serono, Sanofi, Roche, Kyowa Kirin, and travel support from Bristol-Myers Squibb, and Pierre-Fabre. UL declares advisory board honoraria from MSD, Roche, Sanofi, Novartis, Sun Pharma, Almirall Hermal. TE declares consulting fees from BMS, Novartis, Roche, Pierre Fabre, Sanofi; board membership and payment for lectures in speakers bureau from Pierre Fabre, MSD, Roche, BMS, Novartis and Sanofi. CL reports advisory board honoraria from MSD, BMS, Roche, Pierre Fabre, Novartis, Sun Pharma, Sanofi, Kyowa Kirin, Almiral Hermal, Biontech, Merc; speakers fee from MSD, BMS, Roche, Pierre Fabre, Novartis, Sun Pharma, Sanofi, Merck; travel reimbusment from MSD, BMS, Roche, Pierre Fabre, Novartis, Sun Pharma, Sanofi, Kyowa Kirin, Almiral Hermal, Biontech and Merck. JCH declares research support from Bristol Myers Squibb; advisory board honoraria from Pierre Fabre, Sanofi, Sun Pharma and Merck Sharp \& Dome; speakers honoraria from Bristol Myers Squibb, Merck Sharp \& Dohme, Novartis, Roche, Sanofi and Almirall and travel support from Pierre Fabre. TG reports receiving speakers and/or advisory board honoraria from BMS, Sanofi-Grenzyme, MSD, Novartis Pharma, Roche, Abbvie, Almirall, Janssen Lilly, Pfizer, Pierre Fabre; speakers and/or advisory board honoraria from BMS, Sanofi-Grenzyme, MSD, Novartis, Pharma, Roche, Abbvie, Almirall, Janssen Lilly, Pfizer, Pierre Fabre. SH declares advisory boards honoraria from Pierre Fabre, MSD, BMS, Novartis, Sanofi. PM reports board membership and payment for lectures in speakers bureau from Pierre Fabre, GSK, MSD, Merck Germany, Roche, BMS, Novartis, Sanofi. P. Mohr reports research support (to institution): Bristol-MyersSquibb, Novartis, MSD. Honoraria for lectures (personally): Pierre Fabre, GSK, MSD, Merck Germany, Roche, BMS, Novartis, Sanofi, Amgen, SUN-Pharma, Roche Pharma, Bristol-MyersSquibb, Novartis, MSD, Almirall-Hermal, Amgen, Merck-Serono, Bayer, Pierre-Fabre, Sanofi. Honoraria for advisory boards: Pierre Fabre, GSK, MSD, Merck Germany, Roche, BMS, Novartis, Sanofi, Beiersdorf, Almiral-Hermal, AmgenBayersdorf, Roche Pharma, Bristol-MyersSquibb, Novartis, MSD, Almirall-Hermal, Amgen, Pierre-Fabre, Merck-Serono, SUN-Pharma, SUN, Merck-Serono, Sanofi. CP received honoraria (speaker honoraria or honoraria as a consultant) and travel support from Novartis, BMS, Roche, Merck Serono, MSD, Celgene, AbbVie, AMGEN, SUNPHARMA, Allergy Therapeutics and LEO. LMH served as consultant and/or has received honoraria from Amgen, BMS, Curevac, MSD, Novartis, Pierre-Fabre, Roche, Sanofi and Sun Pharma, outside the submitted work. Research funding to institution: Novartis. R. Gutzmer reports research support from Pfizer, Johnson \& Johnson, Novartis, Amgen, MerckSerono, SUN Pharma; honoraria for lectures from Roche Pharma, Bristol-MyersSquibb, Novartis, MSD, Almirall-Hermal, Amgen, Merck-Serono, SUN, Pierre-Fabre, Sanofi, SUN Pharma, Bayer; honoraria for advice from Roche Pharma, Bristol-MyersSquibb, Novartis, 
MSD, Almirall-Hermal, Amgen, Pierre-Fabre, Merck-Serono, 4SC, Incyte, SUN Pharma, Sanofi, Pfizer. JSU is on the advisory board or has received honoraria and travel support from Amgen, Bristol Myers Squibb, GSK, LeoPharma, Merck Sharp and Dohme, Novartis, Pierre Fabre, Roche, Sanofi outside the submitted work. $\mathrm{H}$-US is an employee of Targos Molecular Pathology Inc. and reports research support from Novartis Oncology and received honoraria from MSD, BMS, Roche Pharma, Novartis Oncology, AstraZeneca, Eisai, Takeda, Molecular Health, outside of the submitted work. DS reports personal fees from Amgen, GSK, BMS, Novartis, Roche, Merck, Astra Zeneca, Merck-Serono, Pfizer, Incyte, Array Pierre Fabre, Sanofi Genzyme, Regeneron, 4Sc, InFlaRx, Neracare, Ultimovacs, SunPharma, Philogen, Immunocore, Sandoz-Hexal outside the submitted work. JCB is receiving speaker's bureau honoraria from Amgen, Pfizer, MerckSerono, Recordati and Sanofi; is a paid consultant/advisory board member/DSMB member for Almirall, Boehringer Ingelheim, InProTher, ICON, MerckSerono, Pfizer, 4SC, and Sanofi/Regeneron. His group receives research grants from Bristol-Myers Squibb, Merck Serono, HTG, IQVIA, and Alcedis.

Patient consent for publication Not applicable.

Ethics approval This study was approved by Ethics committee of the University Duisburg-Essen (11-4715; 17-7538-B0) Ethics board of Stockholm (Dnr 2010/1092-31/3)

\section{Provenance and peer review Not commissioned; externally peer reviewed.}

Data availability statement Data are available on reasonable request. All data relevant to the study are included in the article or uploaded as online supplemental information. All the raw data used within this manuscript are available on reasonable request to the corresponding author.

Supplemental material This content has been supplied by the author(s). It has not been vetted by BMJ Publishing Group Limited (BMJ) and may not have been peer-reviewed. Any opinions or recommendations discussed are solely those of the author(s) and are not endorsed by BMJ. BMJ disclaims all liability and responsibility arising from any reliance placed on the content. Where the content includes any translated material, BMJ does not warrant the accuracy and reliability of the translations (including but not limited to local regulations, clinical guidelines, terminology, drug names and drug dosages), and is not responsible for any error and/or omissions arising from translation and adaptation or otherwise.

Open access This is an open access article distributed in accordance with the Creative Commons Attribution Non Commercial (CC BY-NC 4.0) license, which permits others to distribute, remix, adapt, build upon this work non-commercially, and license their derivative works on different terms, provided the original work is properly cited, appropriate credit is given, any changes made indicated, and the use is non-commercial. See http://creativecommons.org/licenses/by-nc/4.0/.

\section{ORCID iDs}

Ivelina Spassova http://orcid.org/0000-0003-4663-7966

Selma Ugurel http://orcid.org/0000-0002-9384-6704

Patrick Terheyden http://orcid.org/0000-0002-5894-1677

Jürgen Christian Becker http://orcid.org/0000-0001-9183-653X

\section{REFERENCES}

1 Becker JC, Stang A, DeCaprio JA, et al. Merkel cell carcinoma. Nat Rev Dis Primers 2017;3:17077.

2 Nghiem PT, Bhatia S, Lipson EJ, et al. PD-1 blockade with pembrolizumab in advanced Merkel-cell carcinoma. N Engl J Med 2016;374:2542-52.

3 Topalian SL, Bhatia S, Amin A, et al. Neoadjuvant nivolumab for patients with resectable Merkel cell carcinoma in the CheckMate 358 trial. J Clin Oncol 2020;38:2476-87.

4 Kacew AJ, Dharaneeswaran H, Starrett GJ, et al. Predictors of immunotherapy benefit in Merkel cell carcinoma. Oncotarget 2020;11:4401-10.

5 Spassova I, Ugurel S, Terheyden P, et al. Predominance of central memory $T$ cells with high T-cell receptor repertoire diversity is associated with response to PD-1/PD-L1 inhibition in Merkel cell carcinoma. Clin Cancer Res 2020;26:2257-67.

6 D'Angelo SP, Russell J, Lebbe C. Efficacy and safety of first-line avelumab treatment in patients with stage IV metastatic Merkel cell carcinoma: a preplanned interim analysis of a clinical trial. JAMA Oncol 2018;4:1-5.
7 Eisenhauer EA, Therasse P, Bogaerts J, et al. New response evaluation criteria in solid tumours: revised RECIST guideline (version 1.1). Eur J Cancer 2009;45:228-47.

8 Shen R, Postow MA, Adamow M, et al. LAG-3 expression on peripheral blood cells identifies patients with poorer outcomes after immune checkpoint blockade. Sci Trans/ Med 2021;13:eabf5107.

9 Bürkner P-C, Vuorre M. Ordinal regression models in psychology: a tutorial. Adv Methods Pract Psychol Sci 2019;2:77-101.

10 Thomas DC. Introduction: Bayesian models and Markov chain Monte Carlo methods. Genet Epidemiol 2001;21:S660-1.

11 Bürkner PC. Advanced Bayesian multilevel modeling with the R package brms. $R$ Journal 2018;180:1-15.

12 Bürkner PC. brms: an R package for Bayesian multilevel models using Stan. J Stat Softw 2017;80:1-28.

13 Buuren Svan, Groothuis-Oudshoorn K. mice : Multivariate Imputation by Chained Equations in R. J Stat Softw 2011;45:1-67.

14 Collins GS, Reitsma JB, Altman DG, et al. Transparent reporting of a multivariable prediction model for individual prognosis or diagnosis (TRIPOD): the TRIPOD statement. Ann Intern Med 2015;162:55-63.

15 Henson SM, Riddell NE, Akbar AN. Properties of end-stage human T cells defined by CD45RA re-expression. Curr Opin Immunol 2012;24:476-81.

16 Walker JW, Lebbé C, Grignani G, et al. Efficacy and safety of avelumab treatment in patients with metastatic Merkel cell carcinoma: experience from a global expanded access program. $J$ Immunother Cancer 2020;8:e000313.

17 Kugel CH, Douglass SM, Webster MR, et al. Age correlates with response to anti-PD1, reflecting age-related differences in intratumoral effector and regulatory T-cell populations. Clin Cancer Res 2018;24:5347-56.

18 Johnstone J, Parsons R, Botelho F, et al. T-Cell phenotypes predictive of frailty and mortality in elderly nursing home residents. $J$ Am Geriatr Soc 2017;65:153-9.

19 De Maeyer RPH, Chambers ES. The impact of ageing on monocytes and macrophages. Immunol Lett 2021;230:1-10.

20 Knepper TC, Montesion M, Russell JS, et al. The genomic landscape of Merkel cell carcinoma and Clinicogenomic biomarkers of response to immune checkpoint inhibitor therapy. Clin Cancer Res 2019;25:5961-71.

21 Lanzavecchia A, Sallusto F. Regulation of T cell immunity by dendritic cells. Cell 2001;106:263-6.

22 Liu Q, Sun Z, Chen L. Memory T cells: strategies for optimizing tumor immunotherapy. Protein Cell 2020;11:549-64.

23 Sallusto F, Geginat J, Lanzavecchia A. Central memory and effector memory T cell subsets: function, generation, and maintenance. Annu Rev Immunol 2004;22:745-63.

24 Im SJ, Hashimoto M, Gerner MY, et al. Defining CD8+ T cells that provide the proliferative burst after PD-1 therapy. Nature 2016;537:417-21.

25 Kurtulus S, Madi A, Escobar G, et al. Checkpoint blockade immunotherapy induces dynamic changes in PD-1-CD8+ tumorinfiltrating T cells. Immunity 2019;50:181-94.

26 Miron M, Kumar BV, Meng W, et al. Human Lymph Nodes Maintain TCF-1 ${ }^{\text {hi }}$ Memory T Cells with High Functional Potential and Clonal Diversity throughout Life. J Immunol 2018;201:2132-40.

27 Sade-Feldman M, Yizhak K, Bjorgaard SL, et al. Defining T cell states associated with response to checkpoint immunotherapy in melanoma. Cell 2018;175:998-1013.

28 Toews K, Grunewald L, Schwiebert S, et al. Central memory phenotype drives success of checkpoint inhibition in combination with CAR T cells. Mol Carcinog 2020;59:724-35.

29 Manjarrez-Orduño N, Menard LC, Kansal S, et al. Circulating T cell subpopulations correlate with immune responses at the tumor site and clinical response to PD1 inhibition in non-small cell lung cancer. Front Immunol 2018;9:1613.

30 Siddiqui I, Schaeuble K, Chennupati V, et al. Intratumoral Tcf1 ${ }^{+}$PD$1^{+} \mathrm{CD}^{+} \mathrm{T}$ Cells with Stem-like Properties Promote Tumor Control in Response to Vaccination and Checkpoint Blockade Immunotherapy. Immunity 2019;50:195-211.

31 Mogilenko DA, Shpynov O, Andhey PS, et al. Comprehensive Profiling of an Aging Immune System Reveals Clonal GZMK ${ }^{+}$ $\mathrm{CD}^{+} \mathrm{T}$ Cells as Conserved Hallmark of Inflammaging. Immunity 2021:54:99-115.

32 Farah M, Reuben A, Spassova I, et al. T-Cell repertoire in combination with T-cell density predicts clinical outcomes in patients with Merkel cell carcinoma. J Invest Dermatol 2020;140:2146-56. 